\title{
Hydrothermal Breakdown of Flexible Metal-Organic Frameworks: A Study by First-Principles Molecular Dynamics
}

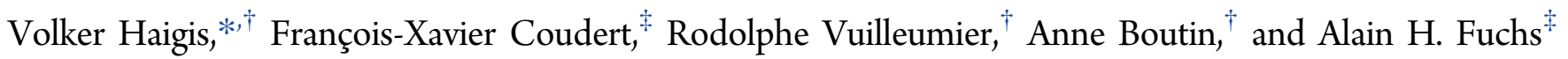 \\ †École Normale Supérieure, PSL Research University, Département de Chimie, Sorbonne Universités - UPMC Univ Paris 06, CNRS \\ UMR 8640 PASTEUR, 24 rue Lhomond, 75005 Paris, France
}

${ }^{\ddagger}$ PSL Research University, Chimie ParisTech - CNRS, Institut de Recherche de Chimie Paris, 75005 Paris, France

Supporting Information

ABSTRACT: Flexible metal-organic frameworks, also known as soft porous crystals, have been proposed for a vast number of technological applications, because they respond by large changes in structure and properties to small external stimuli, such as adsorption of guest molecules and changes in temperature or pressure. While this behavior is highly desirable in applications such as sensing and actuation, their extreme flexibility can also be synonymous with decreased stability. In particular, their performance in industrial environments is limited by a lack of stability at elevated temperatures and in the presence of water. Here, we use first-principles molecular dynamics to study the hydrothermal breakdown of soft porous crystals. Focusing on the material MIL-53(Ga), we show that the weak point of the structure is the bond between the metal center and the organic linker and elucidate the mechanism by which water lowers the activation free energy for the breakdown. This allows us to propose strategies for the synthesis of MOFs with increased heat and water stability.
$\mathrm{M}_{\mathrm{s}}$ etal-organic frameworks (MOFs) form a rapidly growing family of crystalline materials. They are built from metal centers that are connected by organic linkers to form three-dimensional nanoporous structures. The wealth of organic linker chemistry has paved the way, in past decades, to a huge number of newly synthesized materials. Among these, soft porous crystals (SPCs) ${ }^{1}$ form a subclass of stimulusresponsive materials that exhibit reversible, large-amplitude structural changes when exposed to physical or chemical triggers, such as temperature, pressure, or adsorption/ desorption of guest molecules inside the pores of the material. ${ }^{2,3}$ On one hand, this stimulus-responsive character of SPCs makes them strong candidates for applications such as sensing and actuation, and there is a lot of work in the scientific literature focusing on designing novel flexible materials with targeted properties, or coupling the flexibility with novel stimuli. $^{4-6}$ On the other hand, their flexibility often comes at the cost of reduced stability, which limits their application at the industrial level, e.g., at elevated temperatures or in the presence of moisture or liquid water. The development of flexible yet stable MOFs depends on a better fundamental understanding of degradation mechanisms, opening the way to a rational design of novel materials.

Computational modeling of MOFs in general, and SPCs in particular, has greatly advanced in recent years, revealing the atomic-scale mechanisms that underly the stimuli-responsive behavior of materials, such as phase transitions induced by

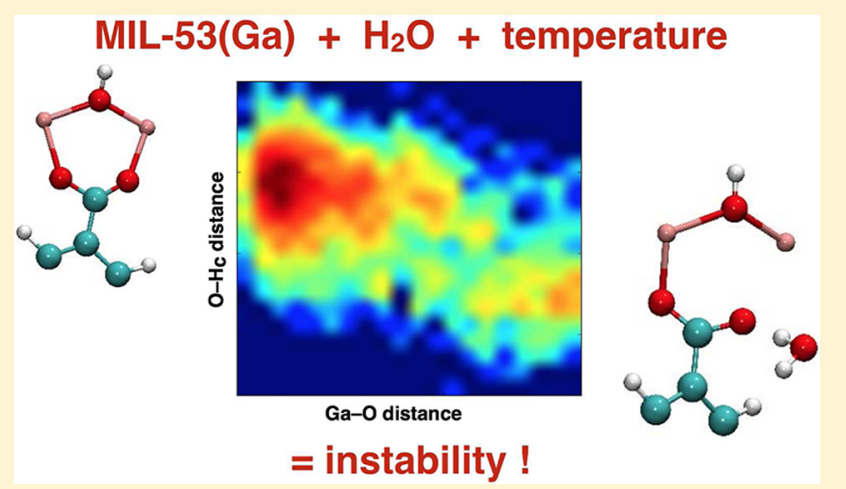

adsorption of guest molecules or mechanical pressure. ${ }^{3,7-9}$ In a recent review, based on experimental results, criteria governing the water stability of MOFs have been proposed. ${ }^{10}$ However, the issue of water stability of MOFs and its microscopic origins has only be addressed by relatively few simulation studies. In a pioneering study, Greathouse and Allendorf reproduced the experimentally observed breakdown of MOF-5 in the presence of water with classical molecular dynamics simulations. ${ }^{11}$ Bellarosa et al. performed classical and first-principles simulations to study the hydrolysis of IRMOFs, identifying the mechanisms by which water attacks the framework and proposing strategies to make the MOF more resistant to water. They found that the basicity of water clusters inside MOFs can serve as a simple descriptor for water stability, thus providing a fast screening tool for candidate materials. ${ }^{12-14}$ De Toni et al. investigated the interplay of hydrophobicity and instability in the presence of water in IRMOF materials by first-principles molecular dynamics simulations. ${ }^{15}$ Another recent paper showed that organic molecules adsorbed in MOF-5 undergo dynamical binding to and release from the metal center. ${ }^{16}$ These studies suggest that the breakdown of the materials is initiated by water bonding to the metal center of the framework and eventually displacing an organic linker. However, these

Received: September 2, 2015

Accepted: October 16, 2015

Published: October 16, 2015 

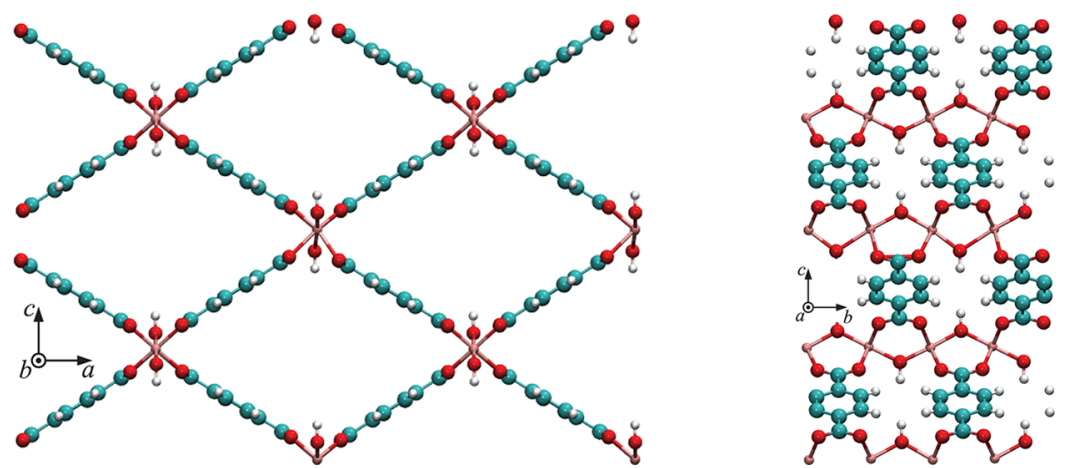

Figure 1. Large-pore structure of MIL-53(Ga), viewed along the diamond-shaped channels (left) and sideview (right). Atomic color code: Ga (pink), O (red), C (turquoise), H (white).

studies focused mostly on the IRMOF family of materials, which are unstable in the presence of water at ambient temperature. There have been, to our knowledge, no theoretical studies of hydrothermal stability at high temperature or concerning highly flexible materials undergoing structural transitions.

As a challenging test case, we study here the hydrothermal breakdown of the gallium-based SPC MIL-53(Ga). ${ }^{17-19}$ Like the other materials of the MIL-53(M) family ( $\mathrm{M}=\mathrm{Al}, \mathrm{Cr}, \mathrm{Fe}$, $\mathrm{Sc}, . .$.$) , its structure is built from trivalent metal cations that are$ bridged by hydroxyl groups to form infinite $-\mathrm{OH}-\mathrm{Ga}-\mathrm{OH}-$ $\mathrm{Ga}-$ chains along the crystallographic $b$ axis (Figure 1). These inorganic chains are connected to each other by benzenedicarboxylate (bdc) linkers in a way that yields diamond-shaped channels along the $b$ axis. MIL-53(Ga) displays a rich phase diagram and occurs in several polymorphs, depending on temperature and the amount of adsorbed guest molecules such as water. ${ }^{20,21}$ This study focuses on the large-pore form, which is shown in Figure 1; the other polymorphs are not considered here. It is stable above $500 \mathrm{~K}$, displays extremely soft behavior along the $a$ and $c$ axes, ${ }^{22}$ and undergoes decomposition at approximately $650 \mathrm{~K}$. Furthermore, we hypothesize that upon immersion into liquid water, the material takes on a "superhydrated" state, structurally close to the large-pore form and of the same topology, in analogy to the Cr variant of the MIL-53 family. ${ }^{23,24}$ The Al variant, MIL-53(Al), was even found to survive short exposures to boiling water, although gradual degradation was observed in the course of several hours. ${ }^{25}$ To our knowledge, however, no data exists in the literature on the thermal stability of MIL-53(Ga) in liquid water.

The aim of this paper is to identify the "weak points" of the MIL-53(Ga) structure, and to study the atomic-scale mechanisms that initiate the decomposition of the material at elevated temperatures. Furthermore, we will see how water inside the channels influences the height of the relevant freeenergy barrier and thus the kinetics of hydrothermal breakdown.

The first step in studying the breakdown of MIL-53(Ga) is to determine the weakest chemical bonds in the framework, i.e., those which break first and thus initiate the collapse of the material. Molecular dynamics simulations of the nonhydrated framework were therefore performed at temperatures of 800, 1000 , and $1500 \mathrm{~K}$, with a duration of at least $12.5 \mathrm{ps}$. We chose these relatively high temperatures in order to accelerate kinetics and to observe rare bond-breaking events even at the small time scales accessible to first-principles simulations. In these runs, the simulation box consisted of only one unit cell, containing 4
$\mathrm{Ga}(\mathrm{OH})$ (bdc). In the case of a bond breaking, the periodic boundary conditions lead to a large and probably unrealistic defect density, especially along the short $b$ axis ( $\sim 6.8 \AA$ ). Therefore, the dynamics following the first bond breaking might not exactly represent the real material's behavior. Nevertheless, these simulations are suitable to qualitatively identify the weakest point in the framework from short, spontaneous dynamics.

At $800 \mathrm{~K}$, all chemical bonds remain intact over the whole simulation, and the unit cell fluctuates around its equilibrium structure, which is orthorhombic with a unit cell volume of $(1500 \pm 100) \AA^{3}$ (deviation at $\left.1 \sigma\right)$. The relatively large standard deviation is a result of framework flexibility, which leads to significant volume fluctuations in isobaric-isothermal simulations, unlike in rigid materials. The volume coincides, within uncertainties, with the volume derived from $\mathrm{X}$-ray diffraction experiments above $493 \mathrm{~K}^{18}\left(1479.7 \AA^{3}\right)$, confirming the validity of our simulation protocol (and in particular the choice of the exchange-correlation functional and dispersion corrections $^{26}$ ). At 1000 and $1500 \mathrm{~K}$, however, defects form within a few picoseconds, both starting with a bond breaking between gallium and an oxygen atom of the carboxyl group of the organic linker. Later in the simulations, this is followed by the breaking of the $\mathrm{Ga}-\mathrm{OH}$ bonds that form the inorganic chains of the framework, eventually leading to a totally disordered state. To confirm this visual inspection, the evolution of the average coordination number of $\mathrm{Ga}$ by $\mathrm{O}$ along the simulation is shown in Figure 2. At 1000 and $1500 \mathrm{~K}$, it exhibits a rapid drop from the initial value of 6 , reaching values as low as 4.5 . The bdc linkers themselves remain intact until the end of the simulation even at the highest temperature. $\mathrm{Ga}-\mathrm{O}$ bond breaking is accompanied by a change of the simulation cell geometry from orthorhombic to triclinic of fluctuating shape, departing up to $30^{\circ}$ from the orthorhombic cell shape, which reflects the decomposition of the framework. These results indicate that the structural collapse of MIL$53(\mathrm{Ga})$ at elevated temperatures is triggered by bond breaking between the $\mathrm{Ga}$ centers and organic linkers.

Now that the weakest points of the MIL-53(Ga) framework have been identified, we turn to the energetics of the $\mathrm{Ga}-\mathrm{O}$ bond breaking and to the question of how the presence of water affects it. We focus here on the kinetic stability of the material: as pointed out by Burtch et al., ${ }^{10}$ even a thermodynamically unstable MOF may be useful in various applications if it is kinetically stable due to large free-energy barriers that preclude structural collapse. Experimental values for the temperature at which anhydrous MIL-53(Ga) starts to 


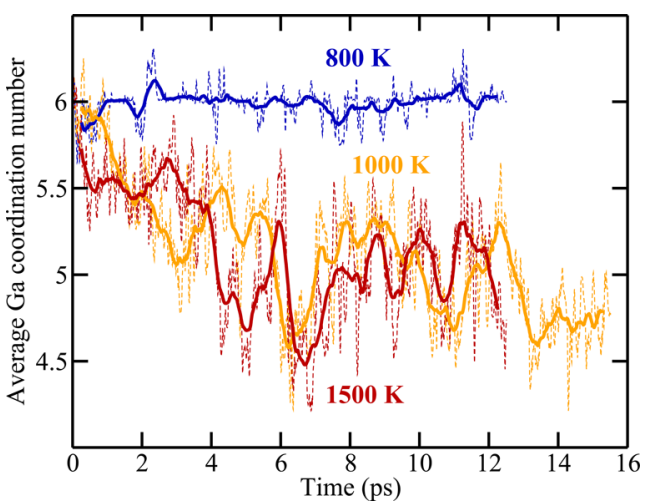

Figure 2. Evolution of the average coordination of $\mathrm{Ga}$ by $\mathrm{O}$ in nonhydrated MIL-53(Ga) at three different temperatures (dashed lines): $800 \mathrm{~K}$ (blue), $1000 \mathrm{~K}$ (orange), and $1500 \mathrm{~K}$ (red). For better visibility, we also plot the running averages over a time interval of 0.5 ps (solid lines). The cutoff distance for $\mathrm{Ga}-\mathrm{O}$ coordination is at $2.8 \AA$, corresponding to the first minimum of the $\mathrm{Ga}-\mathrm{O}$ radial distribution function at $800 \mathrm{~K}$. In order to obtain a continuous evolution in time, we applied a small Fermi-Dirac-like smearing to the cutoff distance.

lose crystallinity range from $613 \mathrm{~K}^{18}$ to $723 \mathrm{~K} \cdot{ }^{17}$ However, in the simulations of the previous section, no bond breaking was observed even at $800 \mathrm{~K}$. This is due to the fact that bond breaking is a rare event at the atomic scale and hence cannot be captured in a statistically meaningful way by equilibrium MD. We therefore turn to the use of an enhanced-sampling technique, well-tempered metadynamics, to study the onset of structural collapse of MIL-53(Ga) at the temperature of 650 K.

This method allowed us to compute the free energy profile along the distance between one $\mathrm{Ga}$ and one $\mathrm{O}$ on the carboxyl group of an organic linker, again with flexible size and shape of the simulation cell. The latter was chosen to contain $1 \times 2 \times 1$ unit cells, i.e., eight $\mathrm{Ga}(\mathrm{OH})$ (bdc) units, in order to reduce the spurious interactions between the defect created during the metadynamics run and its periodic images (the smallest periodic image distance now being larger than $13 \AA$ ). One simulation of 49 ps duration was carried out for the nonhydrated framework (Figure 1), and another of 38 ps duration for a fully hydrated material, containing 48 water molecules inside its pores. We chose this "super-hydrated" form for our purposes in order to highlight in the strongest possible way the effect of water. The superhydrated form is of the same topology as the nonhydrated one, and our simulations yield a slightly larger unit cell volume of $(1660 \pm 20) \AA^{3}$, due to pore opening along the $c$ axis. It has not been reported for MIL$53(\mathrm{Ga})$ in the literature, but its $\mathrm{Cr}$ analogue has been observed after immersion into liquid water. ${ }^{23}$ The number of water molecules in the simulation corresponds to the amount of adsorbed water ( $\sim 6$ water molecules per metal center) determined experimentally for MIL-53(Cr), which has a unit cell volume very simililar to the Ga variant studied here. We suppose that in the hydrated state, the weakest point of the framework is still the metal-linker bond identified in the anhydrous state. This seems reasonable because adsorbed water interacts with the framework only through hydrogen bonding and does not alter the network of covalent bonds.

The resulting free energy profiles along the metadynamics variable (the $\mathrm{Ga}-\mathrm{O}$ distance between metal center and organic linker) are shown in Figure 3. From these profiles, we can accurately determine the activation free energy (i.e., the barrier

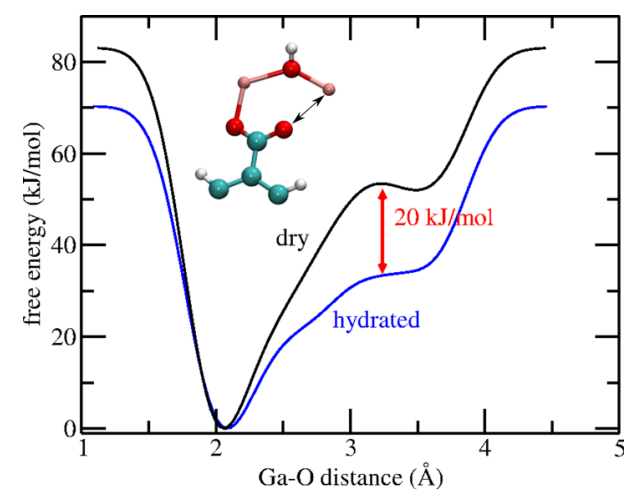

Figure 3. Free energy profiles of $\mathrm{Ga}-\mathrm{O}$ bond breaking for dry (black) and hydrated (blue) MIL-53(Ga) at $650 \mathrm{~K}$. The minimum of each curve has been set to zero. The black arrow in the structure model indicates the metadynamics variable, i.e., the $\mathrm{Ga}-\mathrm{O}$ distance. Atomic color code: Ga (pink), O (red), C (turquoise), H (white). Note that for computational purposes, the sampled $\mathrm{Ga}-\mathrm{O}$ distances were limited to about $3.8 \AA$, hence the free-energy profiles at larger distances are not physical (see text).

height), of the rupture of the $\mathrm{Ga}-\mathrm{O}$ bond, but not the freeenergy difference between the bonded and the dissociated state, because the system was precluded from sampling $\mathrm{Ga}-\mathrm{O}$ distances beyond $\sim 3.8 \AA$. This was done for computational reasons: first, since we are interested in kinetic stability only, we wanted to avoid sampling irrelevant parts of the phase space. Second, in preliminary simulations without restrictions on the $\mathrm{Ga}-\mathrm{O}$ distance, $\mathrm{Ga}-\mathrm{O}$ bond breaking was observed to entail further bond disruptions after a few picoseconds, making it impossible to define in a simple way a unique "broken" state with a single metadynamics variable. With the $\mathrm{Ga}-\mathrm{O}$ distance restricted to below $3.8 \AA$, we made sure that all other bonds remained intact for the duration of the simulation and that we accurately describe the initial step of the hydrothermal instability of MIL-53(Ga). In addition to this study of kinetic stability, we note that the $\mathrm{MD}$ simulations above already showed that the $\mathrm{Ga}-\mathrm{O}$ bond also becomes thermodynamically unstable at high temperatures.

In both the anhydrous and the hydrated case, the minimum of the curve and hence the $\mathrm{Ga}-\mathrm{O}$ equilibrium distance is at $2.07 \AA$, slightly larger than the value of $1.96 \AA$ determined from $\mathrm{X}$-ray diffraction at $293 \mathrm{~K}^{17}$ The difference can be explained by the much higher temperature, $650 \mathrm{~K}$, of the simulations (thermal expansion) and possibly by the tendency of the PBE exchange-correlation functional to overestimate bond lengths by a few percent. In the dry framework, $\mathrm{Ga}-\mathrm{O}$ bond breaking requires overcoming a free-energy barrier of $\Delta G_{\mathrm{dry}}^{+}=53 \mathrm{~kJ} / \mathrm{mol}$ located at a $\mathrm{Ga}-\mathrm{O}$ distance of $3.2 \AA$. This barrier is lowered by $20 \mathrm{~kJ} / \mathrm{mol}$ in the hydrated material, at $\Delta G_{\text {hydrated }}^{\ddagger}=33 \mathrm{~kJ} / \mathrm{mol}$. This indicates that $\mathrm{Ga}-\mathrm{O}$ bond breaking is greatly facilitated by the presence of water, with a kinetic constant for the bond breaking multiplied by a factor of $\exp \left(-\Delta \Delta G^{\ddagger} / R T\right)=40$ in the presence of water. This shows quantitatively how free energy simulations can show the impact of water on the kinetic stability of MIL-53(Ga) at high temperature. We note, in passing, that this free energy barrier in the presence of water is twice that of IRMOF-Oh in the presence of water, with $\Delta G^{\ddagger} / k T$ $=6.1$ here, while $\Delta G^{\ddagger} / k T=3.2$ for IRMOF-0h at low hydration $\left(\Delta F^{\ddagger} \simeq 8 \mathrm{~kJ} / \mathrm{mol}\right.$ at $\left.300 \mathrm{~K}\right){ }^{15}$ This can help explain the difference in behavior between the two materials, where unfunctionalized IRMOF structures are unstable even in the 

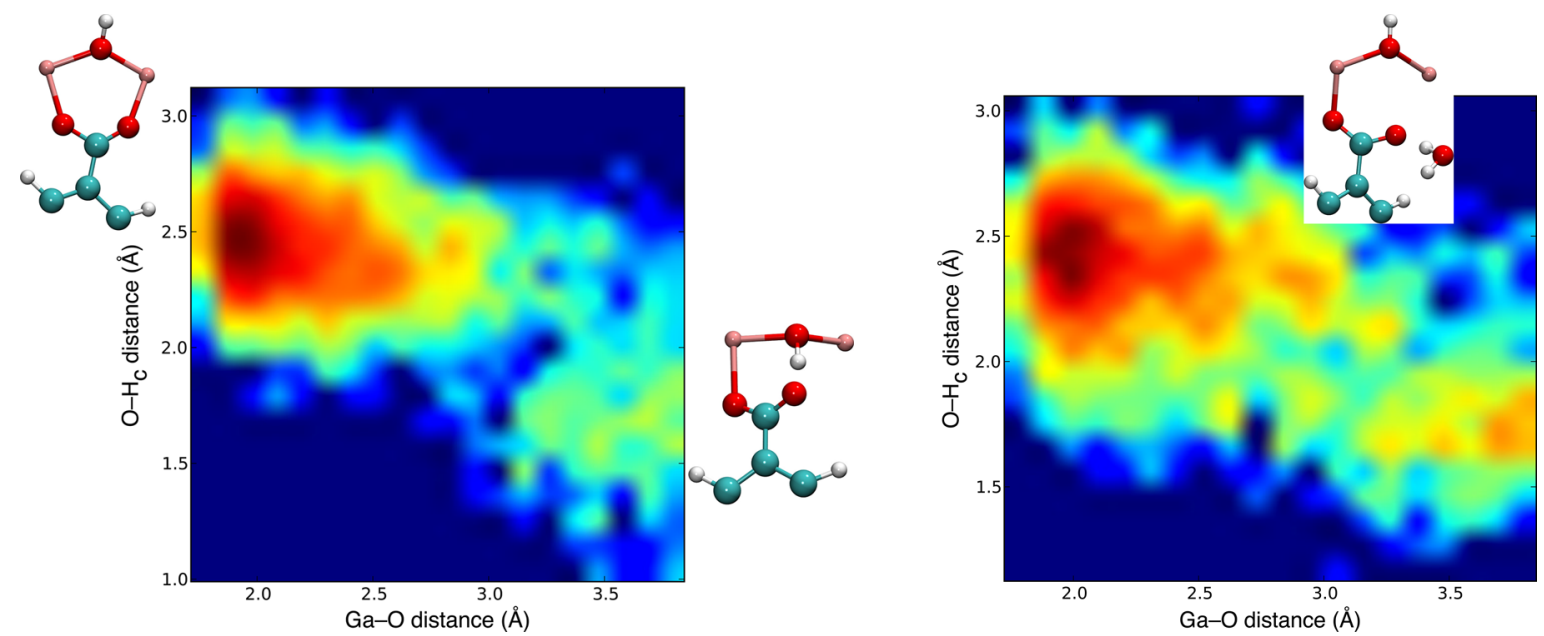

Figure 4. Color-coded histogram describing atomic configurations found during metadynamics simulations of the nonhydrated (left) and the hydrated (right) MIL-53(Ga) framework. The logarithmic color scale ranges from blue (rarely visited) to red (often visited). The abscissa represents the distance between $\mathrm{O}$ on the organic linker and $\mathrm{Ga}$, the ordinate represents the distance between that $\mathrm{O}$ and the closest hydrogen atom $\mathrm{H}_{\mathrm{c}}$. The structure models refer to the configuration in nearby regions of the histograms.

presence of water vapor, while $\mathrm{MIL}-53(\mathrm{Ga})$ is stable in such conditions.

In addition to this quantitative information, atomistic simulations can elucidate in detail at the microscopic level how water facilitates the disruption of metal-linker bonds in MOFs. The most obvious mechanism would be water oxygen attacking the metal site of the framework and eventually displacing the organic linker. This behavior was reported in simulations of hydrated IRMOF structures where adsorbed water directly attacks metal centers, temporarily increasing the coordination of the metal center to form a pentavalent $\mathrm{Zn}^{2+}$ intermediary, before the linker-metal bond is broken to retrieve the tetrahedral coordination. ${ }^{15}$ However, in the case of MIL-53(Ga), our simulations suggest a more complex process. In fact, water oxygen atoms never come closer than $3 \AA$ to the $\mathrm{Ga}$ atom from which the linker is detached. This can be explained by the 6-fold coordination of Ga, which sterically hinders a close water approach. The radial distribution function of water oxygen around this $\mathrm{Ga}$ is very similar to the one of water around the other $\mathrm{Ga}$ atoms that remain in octahedral coordination during the metadynamics simulation (Figure S1). In IRMOF structures, on the other hand, the metal site is only 4-fold coordinated, which allows water to approach it closely. This shows that in MIL-53(Ga), the lowering of the free-energy barrier for metal-linker separation in the hydrated framework is not due to water bonding to the unsaturated metal center after disruption of the metal-ligand bond.

Instead, the oxygen participating in the bond breaking is stabilized by forming hydrogen bonds to surrounding water molecules. This can be seen from Figure 4 where we plot a color-coded histogram of configurations visited during the metadynamics simulations. The two-dimensional configuration space is spanned by the $\mathrm{Ga}-\mathrm{O}$ distance (i.e., the metadynamics variable) and the distance between $\mathrm{O}$ and the closest hydrogen atom, which is termed $\mathrm{H}_{c}$ here and can either be part of the MOF or of a water molecule. The figure visualizes two distinct states of the system: (1) In both the nonhydrated and the hydrated framework, the system spends most of the time in a state with an intact $\mathrm{Ga}-\mathrm{O}$ bond of approximately 2 A length and an $\mathrm{O}-\mathrm{H}_{\mathrm{c}}$ distance around $2.5 \AA . \mathrm{H}_{\mathrm{c}}$ is mostly one of the hydrogen atoms of the BDC linker (see leftmost structure model in Figure 4), but in the hydrated MOF, water hydrogen also contributes. This state corresponds to the minimum of the free-energy curves in Figure 3. (2) The second configuration frequently visited by the systems, both dry and hydrated, features a broken $\mathrm{Ga}-\mathrm{O}$ bond $(\sim 3.5 \AA)$ and an $\mathrm{O}-\mathrm{H}_{c}$ distance close to $1.7 \AA$, implying a hydrogen bond. Here, $\mathrm{H}_{c}$ is mostly the hydrogen atom of a neighboring $\mathrm{OH}$ group of the framework (see middle structure model in Figure 4), but again, in the hydrated MOF, water hydrogen also contributes. This configuration corresponds to the minimum or plateau of the free-energy curves in Figure 3 at $\mathrm{Ga}-\mathrm{O}$ distances beyond $3 \AA$. The main difference between the dry and the hydrated framework in Figure 4 lies in the intermediate region between the two states just described: it is far more frequently visited by the hydrated sytem than by the dry one. It correponds to configurations where the $\mathrm{Ga}-\mathrm{O}$ bond is already broken but the hydrogen bond between $\mathrm{O}$ and the $\mathrm{OH}$ group of the framework has not yet formed. In the nonhydrated system, the dangling $\mathrm{O}$ atom has no bonding partner, leading to an energetically unfavorable situation. In the water-saturated system, however, the $\mathrm{O}$ atoms forms hydrogen bonds with the surrounding water molecules, lowering the energy penalty of $\mathrm{Ga}-\mathrm{O}$ bond breaking (see rightmost structure model in Figure 4).

In conclusion, we have demonstrated in the case of MIL53(Ga) SPCs how first-principles molecular dynamics simulations can help gain microscopic insight and quantitative information about the hydrothermal breakdown of metalorganic frameworks, by combining direct dynamics and free energy methods (in this case, metadynamics). The mechanism for the breakdown of MIL-53(Ga) at high-temperature is initiated by the breaking of the metal-linker bond, which constitutes the weak point of the structure. Subsequently, the linker turns away from $\mathrm{Ga}$, and the dangling $\mathrm{O}$ atom on the linker forms a hydrogen bond with the $\mathrm{OH}$ group of the inorganic $-\mathrm{OH}-\mathrm{Ga}-\mathrm{OH}-$ chain. This prevents the linker from rebonding to Ga quickly. The free-energy barrier for this process was computed to be $53 \mathrm{~kJ} / \mathrm{mol}$, but it is lower by $20 \mathrm{~kJ} /$ mol in the presence of water in the channels of the framework, providing hydrogen bonds to the dangling oxygen. The kinetic thermal stability is therefore expected to decrease significantly 
in the presence of water. The present study also serves as a proof of concept that it is feasible to address the hydrothermal stability of complex materials such as flexible MOFs with firstprinciples methods.

In the perspective of rational design of materials, the atomicscale insights into the structural breakdown of the framework allow us to propose guidelines for synthesizing MIL-53 materials with higher hydrothermal stability. First, and rather obviously, the metal-linker bond should be made as strong as possible, a conclusion that is in line with recommendations reported in the literature. ${ }^{10}$ A possible way to achieve this goal could be to choose more electropositive trivalent metal centers (e.g., Y or Sc). Second, our simulations highlight the destructive role of $\mathrm{OH}$ groups in the inorganic chains of the MIL-53 framework. It should be checked whether these $\mathrm{OH}$ groups can be replaced by $\mathrm{F}$, which would prevent the displaced linker from being stabilized in a distorted configuration by hydrogen bonding. Partially fluoridized MIL-53(Ga) has already been synthesized successfully. ${ }^{17}$ Third, functionalization of the organic linker with small functional groups has been proposed to protect the metal center from a close approach of water molecules. ${ }^{10}$ However, our simulations show that the metal site in MIL-53 is already hardly accessible for water, due its 6-fold coordination. Still, functionalization of the linker might be a promising route, though for a different reason: it could be tuned to prevent the close approach of water to the $\mathrm{O}$ atoms on the carboxyl group. More experimental and computational work is needed to investigate whether these recipes are experimentally feasible and whether such candidate materials would still possess the desirable properties of the MIL-53 family, such as flexibility and adsorption properties. Moreover, while we studied here the large-pore polymorph of MIL$53(\mathrm{Ga})$ in its dry and superhydrated state, it seems worthwhile to investigate, in future work, the interplay between repeated structural transitions of the MIL-53 framework (e.g., in switching applications), different hydration levels and hydrothermal stability.

\section{COMPUTATIONAL METHODS}

The systems were studied by means of density functional theory (DFT)-based molecular dynamics (MD) simulations, using the QUICKSTEP module ${ }^{27}$ of the $\mathrm{CP} 2 \mathrm{~K}$ code package. ${ }^{28}$ In contrast to the few classical force fields available for flexible MOFs, this method allows for an accurate description of bond breaking between the metal centers and organic linkers. Since the soft material is anticipated to undergo massive structural changes in the process, all simulations were performed in the $(N, \sigma, T)$ ensemble with variable size and shape of the simulation cell, following the methodology outlined in previous work. $^{26}$ As pointed out in the literature, ${ }^{11,15}$ simulations at constant volume are not able to fully capture structural degradation, as they artificially impose a given cell geometry. Finally, whereas MD simulations provide full dynamical information about the simulated process, free energies are harder to obtain. Here, we use both "free" MD as well as metadynamics simulations ${ }^{29,30}$ to study the free energy barriers associated with the onset of hydrothermal breakdown. This allows us to quantify the influence of water on the stability of the material at elevated temperatures. Details concerning the computational methods can be found in the Supporting Information.

\section{ASSOCIATED CONTENT}

\section{Supporting Information}

The Supporting Information is available free of charge on the ACS Publications website at DOI: 10.1021/acs.jpclett.5b01926.

Computational methods and radial distribution functions (PDF)

\section{AUTHOR INFORMATION}

\section{Corresponding Author}

*E-mail: volker.haigis@ens.fr.

\section{Notes}

The authors declare no competing financial interest.

\section{ACKNOWLEDGMENTS}

We thank Jean-Pierre Bellat, Jean-Marc Simon, and Marco Salazar for many discussions on this topic. This work was supported by the French Agence Nationale de la Recherche under Project "SOFT-CRYSTAB" (ANR-2010-BLAN-0822$01)$, and performed using high-performance computing resources from GENCI-IDRIS (Grants i2014086114 and x2015087069).

\section{REFERENCES}

(1) Horike, S.; Shimomura, S.; Kitagawa, S. Soft porous crystals. Nat. Chem. 2009, 1, 695-704.

(2) Coudert, F.-X. Responsive Metal-Organic Frameworks and Framework Materials: Under Pressure, Taking the Heat, in the Spotlight, with Friends. Chem. Mater. 2015, 27, 1905-1916.

(3) Schneemann, A.; Bon, V.; Schwedler, I.; Senkovska, I.; Kaskel, S.; Fischer, R. A. Flexible metal-organic frameworks. Chem. Soc. Rev. 2014, 43, 6062-6096.

(4) Bureekaew, S.; Shimomura, S.; Kitagawa, S. Chemistry and application of flexible porous coordination polymers. Sci. Technol. Adv. Mater. 2008, 9, 014108.

(5) Férey, G.; Serre, C. Large breathing effects in three-dimensional porous hybrid matter: facts, analyses, rules and consequences. Chem. Soc. Rev. 2009, 38, 1380-1399.

(6) Czaja, A. U.; Trukhan, N.; Müller, U. Industrial applications of metal-organic frameworks. Chem. Soc. Rev. 2009, 38, 1284-1293.

(7) Odoh, S. O.; Cramer, C. J.; Truhlar, D. G.; Gagliardi, L. Quantum-Chemical Characterization of the Properties and Reactivities of Metal-Organic Frameworks. Chem. Rev. 2015, 115, 6051-6111.

(8) Coudert, F.-X.; Boutin, A.; Fuchs, A. H.; Neimark, A. V. Adsorption Deformation and Structural Transitions in Metal-Organic Frameworks: From the Unit Cell to the Crystal. J. Phys. Chem. Lett. 2013, 4, 3198-3205.

(9) Coudert, F.-X.; Boutin, A.; Jeffroy, M.; Mellot-Draznieks, C.; Fuchs, A. H. Thermodynamic Methods and Models to Study Flexible Metal-Organic Frameworks. ChemPhysChem 2011, 12, 247-258.

(10) Burtch, N. C.; Jasuja, H.; Walton, K. S. Water stability and adsorption in metal-organic frameworks. Chem. Rev. 2014, 114, 10575-10612.

(11) Greathouse, J. A.; Allendorf, M. D. The interaction of water with MOF-5 simulated by molecular dynamics. J. Am. Chem. Soc. 2006, 128, 10678-10679.

(12) Bellarosa, L.; Calero, S.; López, N. Early stages in the degradation of metal-organic frameworks in liquid water from firstprinciples molecular dynamics. Phys. Chem. Chem. Phys. 2012, 14, $7240-7245$.

(13) Bellarosa, L.; Castillo, J. M.; Vlugt, T. J. H.; Calero, S.; López, N. On the Mechanisms Behind the Instability of Isoreticular MetalOrganic Frameworks (IRMOFs) in Humid Environments. Chem. Eur. J. 2012, 18, 12260-12266.

(14) Bellarosa, L.; Gutiérrez-Sevillano, J. J.; Calero, S.; López, N. How ligands improve the hydrothermal stability and affect the 
adsorption in the IRMOF family. Phys. Chem. Chem. Phys. 2013, 15, 17696-17704.

(15) De Toni, M.; Jonchière, R.; Pullumbi, P.; Coudert, F.-X.; Fuchs, A. H. How Can a Hydrophobic MOF be Water-Unstable? Insight into the Hydration Mechanism of IRMOFs. ChemPhysChem 2012, 13, 3497-3503.

(16) Brozek, C. K.; Michaelis, V. K.; Ong, T.-C.; Bellarosa, L.; López, N.; Griffin, R. G.; Dinca, M. Dynamic DMF binding in MOF-5 enables the formation of metastable colbalt-substituted MOF-5 analogues. ACS Cent. Sci. 2015, 1, 252-260.

(17) Vougo-Zanda, M.; Huang, J.; Anokhina, E.; Wang, X.; Jacobson, A. J. Tossing and turning: guests in the flexible frameworks of metal(III) dicarboxylates. Inorg. Chem. 2008, 47, 11535-11542.

(18) Volkringer, C.; Loiseau, T.; Guillou, N.; Férey, G.; Elkaïm, E.; Vimont, A. XRD and IR structural investigations of a particular breathing effect in the MOF-type gallium terephthalate MIL-53(Ga). Dalton Trans. 2009, 2241-2249.

(19) Chaplais, G.; Simon-Masseron, A.; Porcher, F.; Lecomte, C.; Bazer-Bachi, D.; Bats, N.; Patarin, J. IM-19: a new flexible microporous gallium based-MOF framework with pressure- and temperaturedependent openings. Phys. Chem. Chem. Phys. 2009, 11, 5241-5245.

(20) Bousquet, D.; Coudert, F.-X.; Fossati, A. G. J.; Neimark, A. V.; Fuchs, A. H.; Boutin, A. Adsorption induced transitions in soft porous crystals: An osmotic potential approach to multistability and intermediate structures. J. Chem. Phys. 2013, 138, 174706.

(21) Coudert, F.-X.; Ortiz, A. U.; Haigis, V.; Bousquet, D.; Fuchs, A. H.; Ballandras, A.; Weber, G.; Bezverkhyy, I.; Geoffroy, N.; Bellat, J.P.; et al. Water Adsorption in Flexible Gallium-Based MIL-53 MetalOrganic Framework. J. Phys. Chem. C 2014, 118, 5397-5405.

(22) Ortiz, A. U.; Boutin, A.; Fuchs, A. H.; Coudert, F.-X. Anisotropic Elastic Properties of Flexible Metal-Organic Frameworks: How Soft are Soft Porous Crystals? Phys. Rev. Lett. 2012, 109, 195502.

(23) Guillou, N.; Millange, F.; Walton, R. I. Rapid and reversible formation of a crystalline hydrate of a metal-organic framework containing a tube of hydrogen-bonded water. Chem. Commun. 2011, 47, 713-715.

(24) Haigis, V.; Coudert, F.-X.; Vuilleumier, R.; Boutin, A. Investigation of structure and dynamics of the hydrated metal-organic framework MIL-53(Cr) using first-principles molecular dynamics. Phys. Chem. Chem. Phys. 2013, 15, 19049-19056.

(25) Bezverkhyy, I.; Ortiz, G.; Chaplais, G.; Marichal, C.; Weber, G.; Bellat, J.-P. MIL-53(Al) under reflux in water: Formation of $\gamma$ $\mathrm{AlO}(\mathrm{OH})$ shell and $\mathrm{H} 2 \mathrm{BDC}$ molecules intercalated into the pores. Microporous Mesoporous Mater. 2014, 183, 156-161.

(26) Haigis, V.; Belkhodja, Y.; Coudert, F.-X.; Vuilleumier, R.; Boutin, A. Challenges in first-principles NPT molecular dynamics of soft porous crystals: A case study on MIL-53(Ga). J. Chem. Phys. 2014, 141, 064703.

(27) VandeVondele, J.; Krack, M.; Mohamed, F.; Parrinello, M.; Chassaing, T.; Hutter, J. Quickstep: fast and accurate density functional calculations using a mixed Gaussian and plane waves approach. Comput. Phys. Commun. 2005, 167, 103-128.

(28) $\mathrm{CP} 2 \mathrm{~K}$ developers group, CP2K is freely available from www. cp2k.org.

(29) Laio, A.; Parrinello, M. Escaping free-energy minima. Proc. Natl. Acad. Sci. U. S. A. 2002, 99, 12562-12566.

(30) Barducci, A.; Bussi, G.; Parrinello, M. Well-tempered metadynamics: a smoothly converging and tunable free-energy method. Phys. Rev. Lett. 2008, 100, 020603. 